
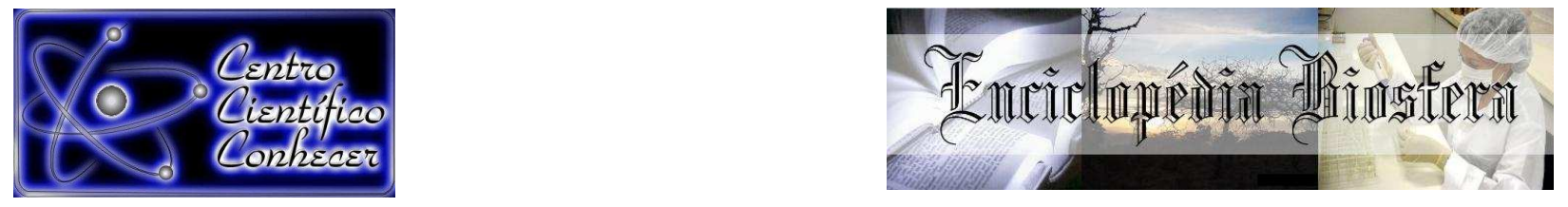

\title{
ASPECTOS CLÍNICOS E EPIDEMIOLÓGICOS DA TUBERCULOSE NO MUNICÍPIO DE IMPERATRIZ, MARANHÃO
}

$\underline{\text { Ivone Pereira da Silva }}{ }^{1}$, Altem Nascimento Pontes ${ }^{2}$, Bruno Costa Silva ${ }^{3}$, lanara Pereira da Silva ${ }^{4}$, Cléa Nazaré Carneiro Bichara ${ }^{5}$

1 Mestranda do Programa de Pós-Graduação em Ciências Ambientais da Universidade do Estado do Pará - UEPA. Belém-PA, Brasil, e-mail:

ivonepds@hotmail.com

2 Doutor em Ciências Físicas. Docente e Pesquisador do Programa de Mestrado em Ciências Ambientais da Universidade do Estado do Pará - UEPA, Belém-PA, Brasil.

3 Graduando de Enfermagem da Universidade Federal do Maranhão - UFMA, Imperatriz, Maranhão.

4 Enfermeira graduada pela Universidade Federal do Maranhão - UFMA, Imperatriz, Maranhão.

5 Médica. Professora adjunta da Universidade do Estado do Pará - UEPA e da Universidade Federal do Pará - UFPA, Belém-PA, Brasil.

Recebido em: 03/10/2016 - Aprovado em: 21/11/2016 - Publicado em: 05/12/2016 DOI: 10.18677/EnciBio_2016B_128

\section{RESUMO}

A tuberculose é uma doença infecciosa que ainda provoca grande impacto social. Apesar dos dados epidemiológicos disponíveis nos sistemas de informação do Ministério da Saúde, ainda há poucos estudos que os analisem regionalmente, de modo a permitir o planejamento de novas estratégias de intervenção, bem como, avaliar as ações que vêm sendo desenvolvidas. Objetivou-se descrever o perfil clínico e epidemiológico dos casos de tuberculose notificados no período de 2009 a 2013 do município de Imperatriz, Maranhão. O estudo foi feito a partir dos casos notificados no Sistema de Informações de Agravos de Notificações. Trata-se de estudo epidemiológico, descritivo, de natureza quantitativa. Dos 364 casos notificados, houve maior incidência da doença entre homens $(65,7 \%)$. Quanto à escolaridade, analfabetos e ensino fundamental representaram $71,0 \% \quad(p=0,0464)$ dos casos. A faixa etária predominante foi de 20 a 39 anos (42,0\%). O principal tipo de entrada se deu através de casos novos (85,7\%). A forma clínica da doença de $89,8 \%$ das notificações foi a pulmonar. Houve associação da tuberculose principalmente com o diabetes $(32,7 \%)$ e com a infecção pelo HIV $(31,8 \%)$. O Tratamento Diretamente Observado foi realizado em $58,5 \%$ dos casos e a alta por cura ocorreu em $88,7 \%$ das notificações. O presente estudo revelou que é necessário maior intensificação à prevenção e ações de vigilância epidemiológica. $O$ aprimoramento do sistema de informações sobre a doença, melhor integração dos programas de controle da tuberculose e Aids e estratégias que viabilizem maior acesso dos homens aos serviços de saúde, assim como dos grupos de maior risco.

PALAVRAS-CHAVE: perfil de saúde, sistemas de informação em saúde, tuberculose. 


\title{
CLINICAL AND EPIDEMIOLOGICAL ASPECTS OF TUBERCULOSIS IN THE MUNICIPALITY OF IMPERATRIZ, MARANHÃO
}

\begin{abstract}
Tuberculosis is an infectious disease that still causes great social impact. Although the epidemiological data available in the information systems of the Ministry of Health, there are few studies that analyze the regional level, in order to allow the planning of new intervention strategies, as well as to assess the actions that are being developed. This study aimed to describe the clinical and epidemiological profile of tuberculosis cases notified in the period 2009 to 2013 in the city of Imperatriz, Maranhão. The study was based on the cases notified in the Information System of Notifiable Diseases. This is an epidemiological, descriptive study of quantitative nature. Of the 364 notified cases, there was greater incidence of disease among men (65.7\%). Regarding education, illiteracy and elementary school represented $71.0 \%$ $(p=0.0464)$ of the cases. The predominant age range was $20-39$ the year $(42.0 \%)$. The main type of entry was through new cases $(85.7 \%)$. the clinical form of the disease of the $89.8 \%$ of the notifications was pulmonary. There was tuberculosis association especially with diabetes (32.7\%) and HIV infection (31.8\%). The Directly Observed Treatment was Held in $58.5 \%$ of cases and a high healing occurred in $88.7 \%$ of notifications. The present study reveals it is most necessary to intensify the prevention and epidemiological surveillance activities. The improvement of information about the disease system, better integration of Tuberculosis and Aids control programs and strategies that enable greater access of men to health services, as well as the higher risk groups.
\end{abstract}

KEYWORDS: health profile, health information systems, tuberculosis.

\section{INTRODUÇÃO}

A tuberculose (TB) é uma doença infecciosa que, embora seja uma das doenças mais antigas de que se tem relato, ainda provoca grande impacto social, constituindo-se em relevante problema de saúde pública, onde fatores sócioculturais contribuem para a carga da doença (SILVA et al., 2014; SAGILI et al., 2016). A nível mundial, atualmente é responsável pelo maior número de óbitos quando se considera um único agente infeccioso (COUTINHO et al., 2012).

A Organização Mundial da Saúde (OMS) estima que um terço da população mundial esteja infectada pelo Mycobacterium tuberculosis e em risco de desenvolver a doença (COELHO et al., 2010). O Brasil faz parte desse grupo, ocupando a 16 posição em número absoluto de casos (BRASIL, 2015a), estando entre os 22 países que concentram cerca de $80 \%$ dos casos de tuberculose (ONOZAKI et al., 2015). Em 2013, a região Norte, Sudeste e Nordeste apresentaram os mais altos coeficientes de incidência: 45,2, 37,1 e 34,7/100 mil habitantes, respectivamente, e entre os Estados nordestinos, o Maranhão aparece em sexto lugar (BRASIL, 2014a).

Entre as principais causas para o agravamento do panorama da tuberculose estão a pobreza, a baixa escolaridade, situações de confinamento, pessoas vivendo em situações de rua, abuso de drogas, a epidemia de AIDS, o processo de envelhecimento da população, os intensos movimentos migratórios e o crescimento demográfico desordenado, além das dificuldades de operacionalização dos programas de controle da tuberculose (CALIARI \& FIGUEIREDO, 2012).

A OMS estabeleceu metas desafiadoras para os países em sua estratégia 
pós-2015 (BRASIL, 2015a). A nova estratégia da OMS, de projetar seu foco de ação no longo prazo (2016-2035), já provocou a adoção de mudanças importantes e outras que deverão efetuar-se nos serviços de saúde dedicados ao atendimento de pessoas com TB (MACIEL, 2016). Nesse contexto, o cenário atual tem exigido a elaboração de estratégias para atingir as metas estabelecidas pelo Ministério da Saúde, como o controle da doença, que necessita compreender aspectos humanitários, econômicos e de saúde pública (SANTOS et al., 2012).

Considerando que os dados epidemiológicos estão disponíveis nos sistemas de informação do Ministério da Saúde, ainda há poucos estudos que os analisem regionalmente, de modo a permitir o planejamento de novas estratégias de intervenção, bem como, avaliar as ações que vêm sendo desenvolvidas (CAVALCANTE \& SILVA, 2013). Assim, haja vista a falta de estudos sobre o perfil da doença no Estado do Maranhão, perbeceu-se a necessidade de analisar os aspectos da doença no município de Imperatriz, um dos municípios prioritários para o controle da tuberculose no Maranhão (SANTOS NETO et al., 2012).

Nessa perspectiva, o presente estudo teve como objetivo descrever o perfil clínico e epidemiológico dos casos de tuberculose notificados à Secretaria Municipal de Saúde, no período de 2009 a 2013, no município de Imperatriz-MA.

\section{MATERIAL E MÉTODOS}

Trata-se de um estudo epidemiológico, descritivo e de natureza quantitativa. Foram coletados dados secundários dos casos de tuberculose ocorridos no município de Imperatriz-MA, entre os anos de 2009 e 2013. A coleta de dados foi realizada no mês de setembro de 2015, junto à Secretaria Municipal de Saúde de Imperatriz. Obteve-se um total de 364 casos de tuberculose baseados nos registros das Fichas de Notificação Compulsória de Tuberculose e Fichas de Investigação de Tuberculose, coletados no banco de dados do Sistema de Informações de Agravos de Notificações (SINAN) do município.

As bases populacionais utilizadas para cálculos foram coletadas em censos e projeções demográficas do Instituto Brasileiro de Geografia e Estatística (IBGE, 2010) referentes ao período do estudo. As variáveis estudadas foram: ano da notificação, sexo, faixa etária, escolaridade, ocupação, doença relacionada ao trabalho, tipo de entrada, forma clínica, situação de encerramento, coinfecção pelo HIV, baciloscopia de escarro, doenças associadas e Tratamento Diretamente Observado (TDO) ou supervisionado.

A população de estudo foi constituída por todos os indivíduos diagnosticados com tuberculose notificados e residentes no município de Imperatriz. As informações coletadas foram organizadas em um banco de dados eletrônico. As variáveis quantitativas foram descritas através de seu valor absoluto, de distribuição de frequências e taxa de incidência, em casos para cada 100 mil habitantes, para cada ano da série histórica investigada. A associação das variáveis selecionadas para caracterizar o perfil epidemiológico e a série histórica estudada, foi verificada a partir do teste Qui-quadrado e do Teste G, considerando o valor de $5 \%(p<0,05)$ para a significância. Essa análise foi realizada no programa BioEstat 5.0.

$\mathrm{O}$ estudo foi realizado respeitando a confidencialidade e o anonimato dos casos notificados no SINAN/NET e a coleta de dados foi realizada após liberação da Secretaria Municipal de Saúde de Imperatriz. Utilizaram-se somente as variáveis aqui apresentadas. O projeto foi aprovado pelo Comitê de Ética em Pesquisa 
Envolvendo Seres Humanos da Universidade do Estado do Pará (UEPA) (parecer número 1.622.428), respeitando os termos da Resolução 466/12 do Conselho Nacional de Saúde.

\section{RESULTADOS}

Durante a série de anos de 2009 a 2013, foram notificados 364 casos de tuberculose à Secretaria Municipal de Saúde de Imperatriz. Constatou-se que 239 $(65,7 \%)$, destes casos, eram do sexo masculino. A faixa etária de 20 a 39 anos foi a mais atingida pela tuberculose $(42,0 \%)$, sendo que a faixa etária de 50 a 69 anos representou $24,1 \%$ dos casos.

Em relação à escolaridade predominaram indivíduos com ensino fundamental $(47,5 \%)$, mostrando-se estatisticamente significativo em toda a série histórica $(p=0,0464)$. Porém 50 registros referentes à escolaridade tiveram o campo ignorado pelo profissional responsável pela notificação. Cerca de $49,0 \%$ dos pacientes eram economicamente ativos - ou seja, contavam com um trabalho formal ou informal -, $44(12,1 \%)$ eram donas de casa, $62(17,0 \%)$ aposentados ou pensionistas e $27(7,4 \%)$ estudantes. Sendo que entre os trabalhadores economicamente ativos, $26,8 \%$ dos registros da doença foram relacionados ao trabalho (Tabela 1).

TABELA 1 - Características sociodemográficas dos casos notificados de tuberculose no Município de Imperatriz, Estado do Maranhão. Brasil, 2009-2013

\begin{tabular}{|c|c|c|c|c|c|c|}
\hline Características & 2009 & 2010 & 2011 & 2012 & 2013 & p-valor \\
\hline Gênero $^{a}$ & & & & & & 0,1779 \\
\hline Masculino & 52 & 44 & 57 & 43 & 43 & \\
\hline Feminino & 34 & 33 & 24 & 18 & 16 & \\
\hline Idade $^{\mathrm{b}}$ & & & & & & 0,3907 \\
\hline Menor de 1 ano & 1 & 2 & 0 & 0 & 0 & \\
\hline 1 a 19 anos & 6 & 6 & 7 & 4 & 3 & \\
\hline 20 a 39 anos & 33 & 28 & 37 & 30 & 25 & \\
\hline 40 a 59 anos & 22 & 24 & 20 & 14 & 22 & \\
\hline 60 anos e mais & 25 & 20 & 17 & 5 & 13 & \\
\hline Escolaridade $^{\mathrm{b}}$ & & & & & & 0,0464 \\
\hline Analfabeto & 13 & 11 & 12 & 11 & 3 & \\
\hline Ensino Fundamental & 40 & 37 & 45 & 22 & 29 & \\
\hline Ensino Médio & 17 & 12 & 14 & 15 & 15 & \\
\hline Ensino Superior & 0 & 5 & 3 & 4 & 6 & \\
\hline Ign/Branco & 16 & 12 & 7 & 9 & 6 & \\
\hline Não se aplica* & 4 & 1 & 0 & 1 & 0 & \\
\hline TB relacionada ao trabalho ${ }^{b}$ & & & & & & 0,3918 \\
\hline Sim & 4 & 5 & 2 & 6 & 2 & \\
\hline Não & 76 & 72 & 79 & 55 & 57 & \\
\hline Ign/Branco & 6 & 0 & 0 & 0 & 0 & \\
\hline
\end{tabular}




\begin{tabular}{|c|c|c|c|c|c|}
\hline \multicolumn{6}{|l|}{ Ocupação ${ }^{b}$} \\
\hline Estudante & 5 & 6 & 7 & 3 & 0 \\
\hline Dona de casa & 10 & 10 & 8 & 11 & 5 \\
\hline Aposentado/Pensionista & 19 & 13 & 13 & 8 & 0 \\
\hline Trabalhador Agropecuário em geral & 2 & 7 & 4 & 2 & \\
\hline Vendedor de comércio varejista & 5 & 1 & 1 & 3 & \\
\hline Pedreiro & 3 & 0 & 5 & 4 & \\
\hline Servente de obras & 1 & 1 & 4 & 0 & \\
\hline Vigilante & 1 & 2 & 3 & 0 & \\
\hline Comerciante varejista & 1 & 1 & 3 & 0 & \\
\hline $\begin{array}{l}\text { Professor de Educação de Jovens e } \\
\text { adultos }\end{array}$ & 1 & 2 & 2 & 0 & \\
\hline Motorista de taxi & 0 & 2 & 1 & 1 & \\
\hline Pintor & 1 & 1 & 1 & 0 & 1 \\
\hline Outras & 24 & 17 & 22 & 17 & 18 \\
\hline
\end{tabular}

Fonte: SMS - Imperatriz, MA - Sistema de Informações de Agravos e de Notificações (SINAN), Divisão de Vigilância Epidemiológica.

*Trata-se de indivíduos que não estão matriculados no sistema formal de educação.

${ }^{a}$ Teste Qui-quadrado ${ }^{\mathrm{b}}$ Teste G

Durante o período estudado, a taxa média anual de incidência foi de 29,5 casos/100 mil habitantes. O número de casos por ano e a taxa de incidência apresentaram valores menores nos anos de 2011 e 2012, e em 2013 constatou-se a maior taxa de incidência (34,2/100 mil habitantes). Foi possível, ainda, observar que o número de registros em Imperatriz correspondeu a menos de $4 \%$ dos casos de tuberculose notificados no Estado do Maranhão (Tabela 2).

TABELA 2 - Frequência dos casos notificados de tuberculose conforme o ano de diagnóstico no Município de Imperatriz, Estado do Maranhão. Brasil, 2009-2013

\begin{tabular}{cccc}
\hline Ano & Número de casos & População & Taxa de incidência (\%) \\
\hline 2009 & 77 & 236.691 & 32,5 \\
2010 & 81 & 247.553 & 32,7 \\
2011 & 61 & 248.805 & 24,5 \\
2012 & 59 & 250.063 & 23,6 \\
2013 & 86 & 251.468 & 34,2 \\
\hline
\end{tabular}

Fonte: SMS - Imperatriz-MA - Sistema de Informações de Agravos e de Notificações (SINAN), Instituto Brasileiro de Geografia e Estatística. 
Em relação aos dados clínicos dos casos de tuberculose estudados, observou-se que do total de casos, $327(89,8 \%)$ foram classificados com a forma pulmonar da doença, independente da idade, enquanto 10,2\% dos casos representaram as formas extrapulmonares da doença. Durante a série de anos, a forma pulmonar manteve-se estável, apresentando uma variação de $94,2 \%$ (2009) a $89,3 \%$ (2013). Do total de 110 casos (30,2\%) com agravos associados, 36 casos $(32,7 \%)$ estavam associados ao diabetes, e $35(31,8 \%)$ casos ao HIV (Figura 1$)$.

FIGURA 1: Porcentagem de casos de tuberculose, por forma da doença, riscos
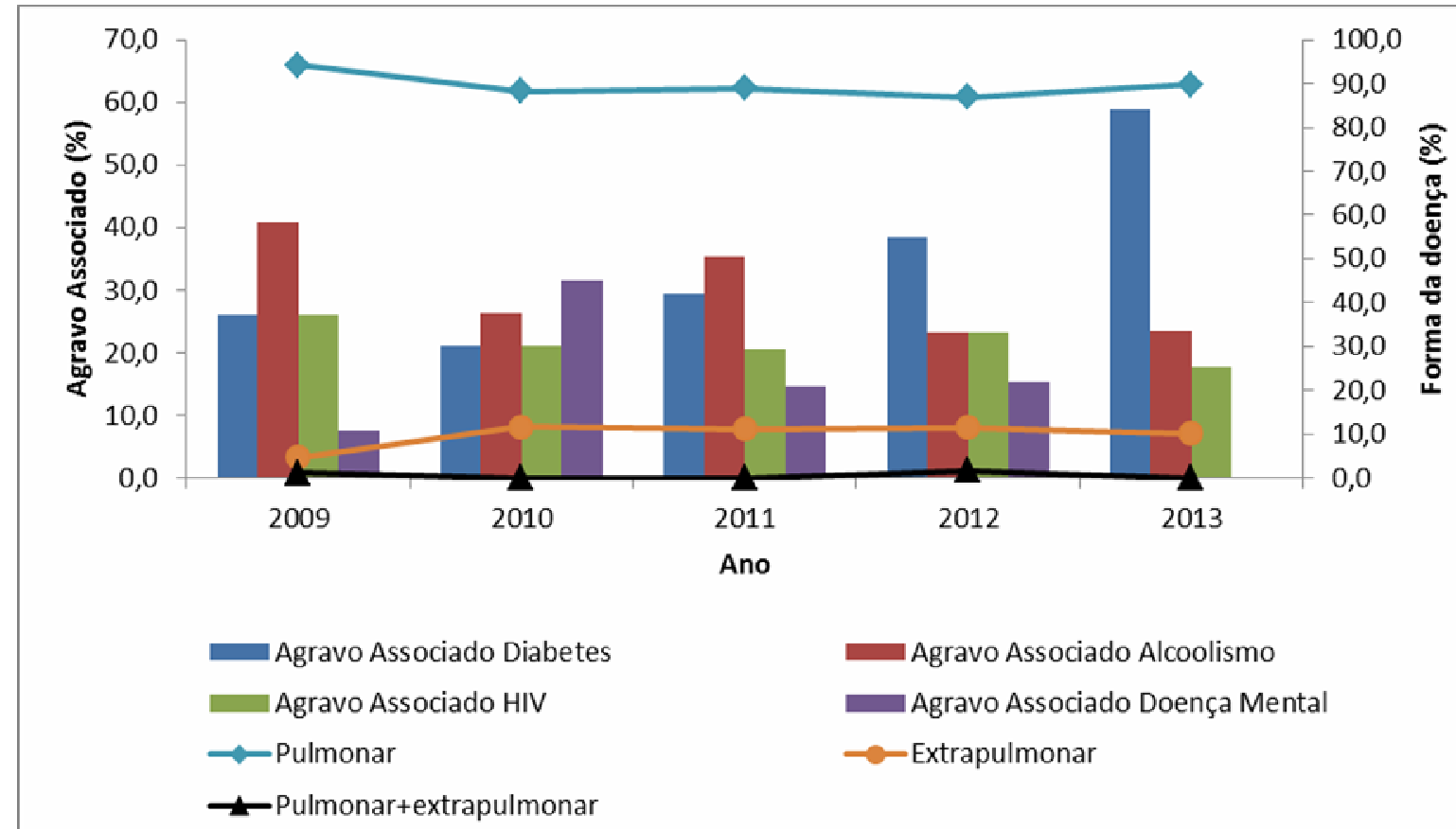

Agravo Associado Alcoolismo

_ Agravo Associado Doença Mental

- Extrapulmonar

associados e ano de diagnóstico, em Imperatriz-MA, entre 2009 e 2013.

Fonte: SMS - Imperatriz-MA - Sistema de Informações de Agravos e de Notificações (SINAN).

Ressalta-se que para as notificações sobre agravos associados, não estavam disponíveis $3,8 \%$ dos dados para diabetes e $6,6 \%$ para doença mental e alcoolismo. Sendo assim, é presumível que esses casos não tenham sido investigados, ou ainda que se refiram a situações onde esses agravos não estavam associados à tuberculose.

Entre os indivíduos que realizaram a sorologia para o HIV, $13,1 \%$ dos resultados foram positivos, ao passo que os não infectados pelo vírus representam $81,5 \%$ das ocorrências e $5,4 \%$ dos casos que realizaram a sorologia ainda se encontram em andamento. Porém, a sorologia não foi realizada em 180 casos do total de pacientes com tuberculose $(49,5 \%)$. 
Relata-se ainda que a baciloscopia, considerado um instrumento fundamental no diagnóstico da tuberculose, foi realizada em $76,1 \%$ dos casos notificados da doença. Do total de exames realizados, os resultados se mostraram positivos e negativos, respectivamente, em $54,2 \%$ e $42,6 \%$. Entre as notificações, $312(85,7 \%)$ entraram como casos novos, 26 (7,1\%) como recidivas, 25 (6,9\%) como transferências e $1(0,3 \%)$ caso de reingresso após abandono (Figura 2$)$.

FIGURA 2: Distribuição dos casos de tuberculose, segundo o tipo de entrada e o

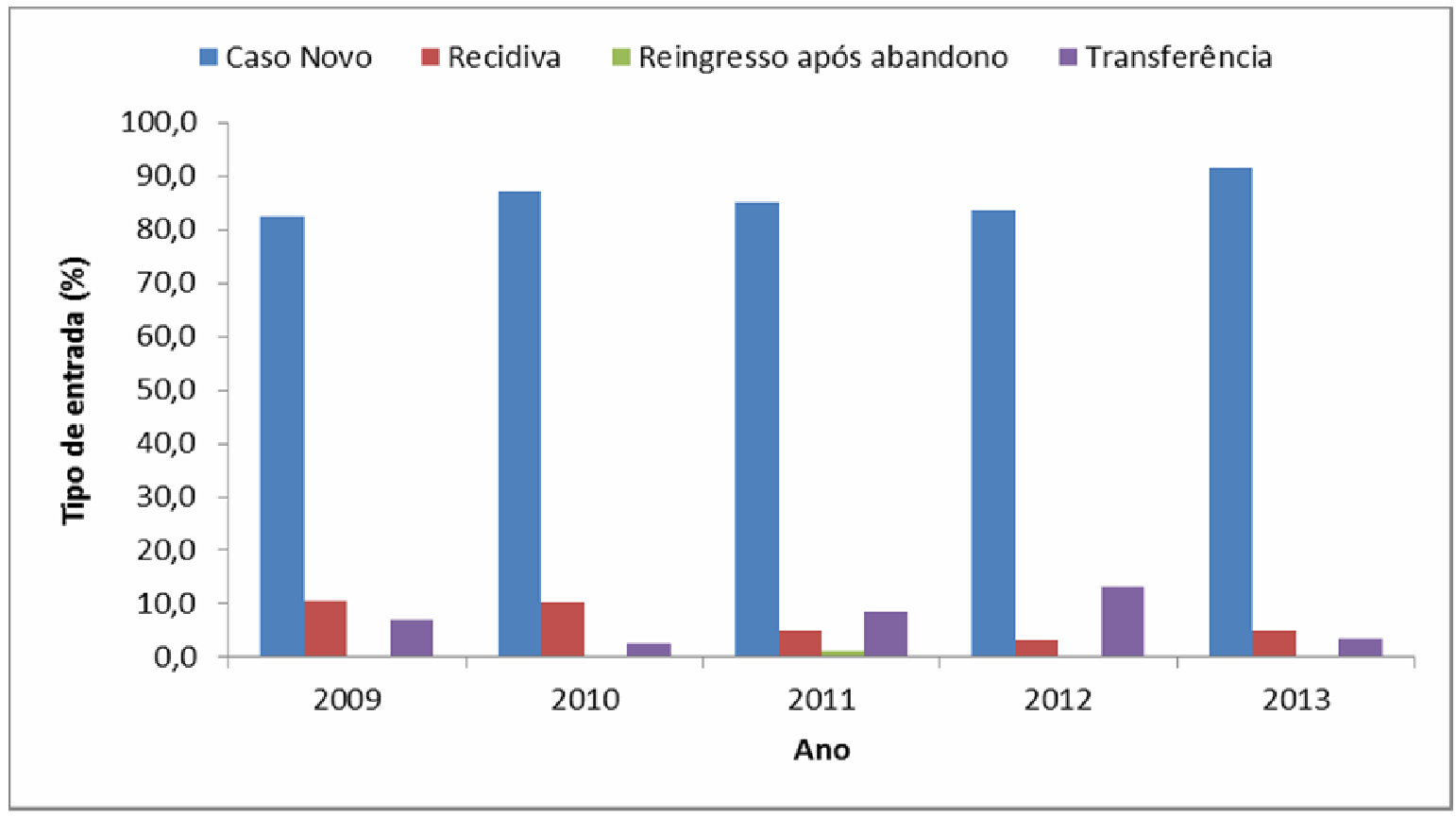

ano de dianóstico, em Imperatriz-MA, entre 2009 e 2013.

Fonte: SMS - Imperatriz-MA - Sistema de Informações de Agravos e de Notificações (SINAN).

Quanto à condição de encerramento dos casos notificados no período de estudo, $323(88,7 \%)$ tiveram alta por cura, $11(3,0 \%)$ abandonaram o tratamento, 6 $(1,6 \%)$ foram transferidos para outros serviços de saúde e $19(5,2 \%)$ foram a óbito. Com relação ao tipo de administração do tratamento, $213(58,5 \%)$ casos se referem ao TDO realizado por algum profissional da saúde da Estratégia Saúde da Família ou também por uma pessoa próxima ao paciente, sendo o responsável pela supervisão do tratamento. O percentual restante se refere ao tratamento autoadministrado (Figura 3). 


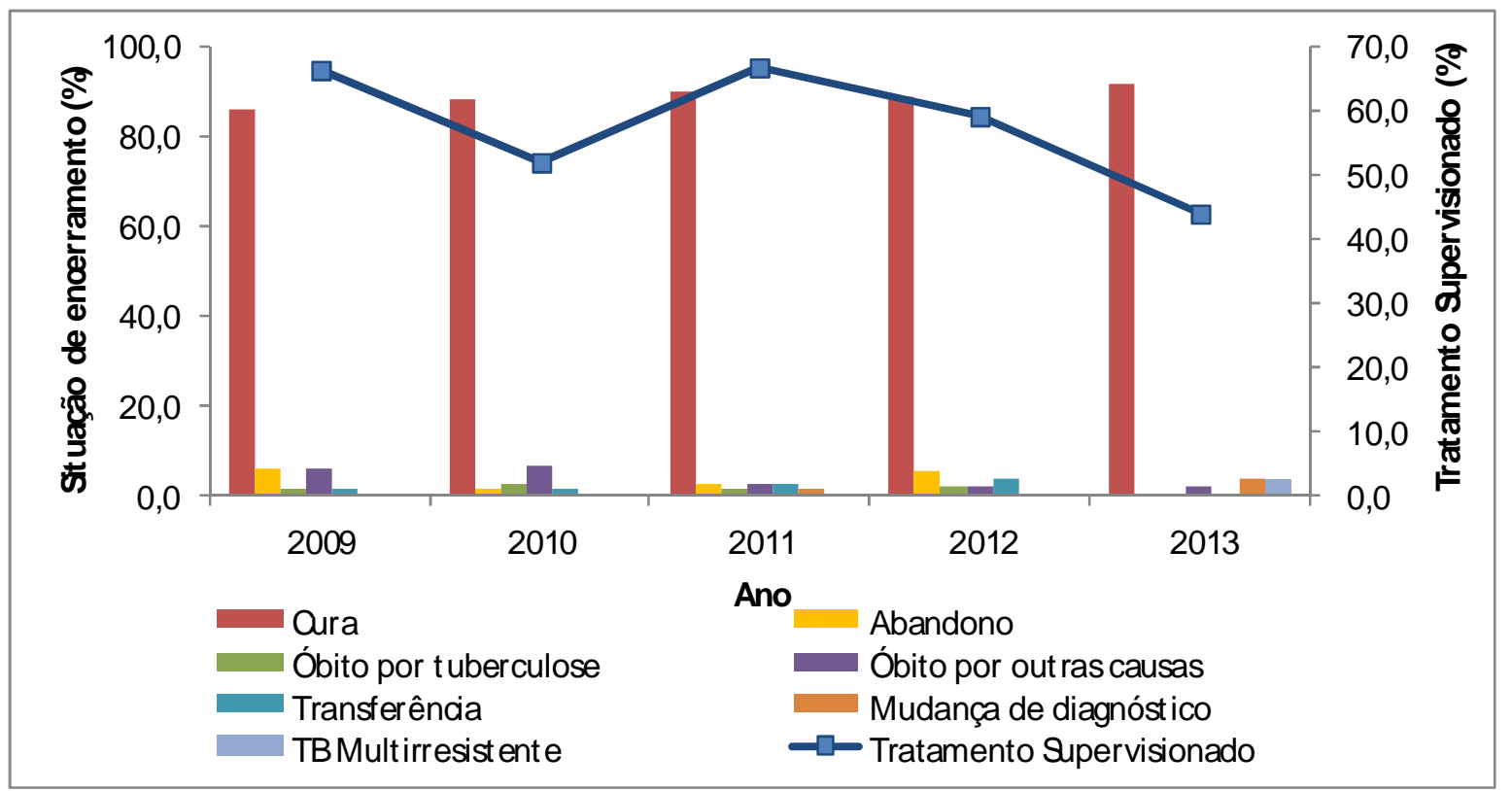

FIGURA 3: Porcentagem de casos de tuberculose por situação de encerramento, tratamento supervisionado e ano de diagnóstico, entre 2009 e 2013, em Imperatriz-MA.

Fonte: SMS - Imperatriz-MA - Sistema de Informações de Agravos e de Notificações (SINAN)

\section{DISCUSSÃO}

Em Imperatriz, durante a série histórica estudada, constatou-se que a tuberculose atingiu mais indivíduos com faixa etária de 20 a 39 anos. Tal resultado é corroborado pelos estudos de SILVA et al., (2014) e DAMACENO et al., (2014), mostrando que a tuberculose acomete mais a classe de adultos jovens, os quais representam o grupo de maior produtividade social, trazendo assim implicações econômicas e sociais para o próprio indivíduo e para a família (DURANS et al., 2013).

A faixa etária de 50 a 69 anos representou $24,2 \%$ dos casos notificados no município. Tal fato constitui-se um problema de saúde pública, diante do crescimento populacional desse grupo, podendo-se notar com isso, que a tuberculose em Imperatriz-MA, assim como no país, tem apresentado elevada incidência em pessoas com idade maior que 50 anos (COELHO et al., 2010).

O aumento da incidência entre esses indivíduos está associado ao aumento da expectativa de vida e também guarda relação com a reativação de focos latentes, devido ao aumento da idade, uma vez que os mecanismos fisiológicos e imunológicos se encontram naturalmente diminuídos e favorecem a instalação da doença, reativada pela ocorrência de desordens crônicas (SILVA et al., 2013).

Em relação à ocupação, resultados semelhantes foram encontrados em estudo realizado em Portugal (LOPES et al., 2011), onde 50,7\% dos casos de tuberculose eram constituídos por uma população ativa. Neste estudo, as pessoas acometidas pela doença têm ocupações diversas, prevalecendo aposentados e donas de casa.

A situação de vulnerabilidade na qual se encontram os aposentados e as 
donas de casa é fator preditor para o acometimento pela tuberculose e parece estar relacionado à baixa renda, que condiciona inadequadas condições de moradia e insalubridade nas atividades domésticas, alimentação inadequada, além de outros fatores como a dificuldade de acesso aos serviços de saúde e a baixa resolutividade da política de atenção ao idoso (CAVALCANTE \& SILVA, 2013).

A incidência da doença no município de Imperatriz relacionada ao trabalho foi de 2,1/100 mil habitantes, semelhante ao coeficiente do Estado e região $(2,1)$ e superior ao do Brasil $(1,3)$ (BRASIL, 2015b). Isso representa um sério problema na esfera socioeconômica do município, pois a doença tende a afastar o trabalhador de suas atividades ocupacionais, aumentando a taxa de absenteísmo e diminuindo a produtividade das empresas (HINO et al., 2011).

Em relação ao predomínio masculino sobre os casos de tuberculose, trabalhos semelhantes (ALVES et al., 2014; BRASIL, 2014b) referem que esse resultado se associa ao fato dos homens se exporem com maior frequência aos fatores de risco e procurarem menos os serviços de saúde (PEREIRA et al., 2015). Tal situação suscita algumas reflexões acerca das possíveis lacunas no PNCT, que tem direcionado, em menor proporção, ações mais específicas para alcançar a população mais atingida pela doença (OROFINO et al., 2012).

Verifica-se que a pouca escolaridade estatisticamente significativa $(p=0,0464)$ encontrada neste estudo, foi concordante com a literatura, que relata a inexistência ou baixa escolaridade da maioria dos casos (REIS et al., 2013; CAVALCANTE \& SILVA, 2013).

A baixa escolaridade aumenta a vulnerabilidade à tuberculose e se torna um importante fator responsável pelo aumento da incidência e do abandono ao tratamento, uma vez que pode comprometer a compreensão do tratamento e da doença (OROFINO et al., 2012; ALVES et al., 2014). Assim, as ações de educação em saúde, se tornam um mecanismo que possibilita um maior entendimento e apreensão dos conhecimentos levantados sobre o viver com a doença ou o estar doente (BRASIL, 2010).

É de fundamental o conhecimento do perfil socioeconômico da população acometida pela tuberculose e se faz necessária não apenas para orientar as políticas públicas, mas também para nortear as ações da Atenção Básica, por meio das equipes de Saúde da Família (COELHO et al., 2010).

O município de Imperatriz-MA apresentou, no período analisado, uma taxa de incidência média de 29,5/100 mil habitantes, sendo menor que as encontradas em outros estudos, como em Piripiri-PI (MASCARENHAS et al., 2005) e em São Luís-MA (SILVA et al., 2014), porém maior do que no município de Contagem-MG (ALVES et al., 2014) e do que a taxa estimada para o Brasil, de 25,9/100 mil habitantes para 2015 (BRASIL, 2014a).

As taxas de incidência da doença no município têm se mantido maiores que as do Estado, onde a taxa média foi de 17,7/100 mil habitantes no período compreendido entre 2010 e 2013 (BRASIL, 2015b). Nos últimos cinco anos, esse indicador vem apresentando tendência de queda, semelhante à taxa de incidência do Estado e País, apesar de em 2013 ter se elevado novamente, porém esse estudo não pode demonstrar as quais eventos esse parâmetro está associado. Esses resultados demonstraram que o controle da tuberculose ainda se constitui um desafio para as políticas públicas de saúde em Imperatriz-MA, uma vez que se verifica a persistência da doença no município (MENDONCA \& FRANCO, 2015). 
O perfil dos doentes segundo a forma clínica mostrou predomínio da forma pulmonar $(89,8 \%)$, não se diferenciando da caracterização encontrada em outros estudos epidemiológicos (CALIARI \& FIGUEIREDO, 2012; SILVA et al., 2013; DAMACENO et al., 2014). Enfatiza-se que a forma pulmonar é a que necessita de maior atenção para as estratégias de controle da tuberculose, uma vez que se trata da forma de maior infectividade (REIS et al., 2013). Por isso, é imprescindível que o diagnóstico e o tratamento da tuberculose sejam realizados precocemente, na atenção primária, por considerar a forma pulmonar a de mais fácil diagnóstico e de mais baixo custo, utilizando a busca ativa de casos, de forma a intervir na cadeia de transmissão da doença (HINO et al., 2011).

Entre os casos com agravos associados, observou-se que o diabetes e HIV foram as duas principais comorbidades associadas à tuberculose, o que difere de outros estudos, onde o HIV e alcoolismo foram os principais agravos associados (CAVALCANTE \& SILVA, 2013) e a doença mental foi a mais prevalente no estudo de SILVA et al., (2013).

O diabetes é um fator de risco bem conhecido para o desenvolvimento de tuberculose ativa, elevando o risco em 2 a 4 vezes (BRASIL, 2011). Em 2011, a OMS e a Internacional União contra a Tuberculose e Doenças Pulmonares lançaram um novo quadro de cooperação para o cuidado e controle do diabetes e tuberculose, sendo que uma das atividades mais relevantes é a implementação de rotina de triagem bidirecional das duas doenças, que necessitam ser executadas com maior eficiência (LIN et al., 2012).

Quanto à associação com o HIV, esta é considerada um importante fator para o aumento do abandono do tratamento, uma vez que a ocorrência de interações medicamentosas e os efeitos provocados pelas reações adversas em pacientes coinfectados são maiores quando comparados aqueles não infectados pelo HIV, sendo que, muitas vezes, esses pacientes dão prioridade ao tratamento da AIDS, em detrimento do tratamento da tuberculose (REIS et al., 2013; SILVA et al., 2013).

O teste anti-HIV não foi realizado em um grupo considerável $(49,5 \%)$ de pacientes, diferente dos resultados encontrados em estudo semelhante (ALVES et al., 2014) onde somente $5,7 \%$ dos indivíduos não realizaram a sorologia. Esse fato dificulta o conhecimento do real cenário da coinfecção TB e HIV em Imperatriz. Estudos indicam que isso pode ser explicado pelo fato de o paciente se sentir discriminado ao ser convidado para fazer a sorologia para o HIV, pois mesmo fazendo parte das recomendações do Ministério da Saúde, é necessário solicitar a autorização do paciente para realizá-lo, o qual pode recusá-lo por direito de cidadania (HINO et al., 2011).

Os aspectos epidemiológicos dessa associação representam um grande desafio, pois são consideradas as enormes dificuldades que a saúde pública enfrenta na organização das ações de controle das duas doenças, as quais são executadas por programas distintos, ainda desarticulados e com políticas de controle em níveis diversos de atenção (GUIMARÃES et al., 2012).

Sobre o alcoolismo, assim como em outras dependências químicas, a adesão ao tratamento é prejudicada em decorrência do estilo de vida adotado pelos dependentes (CALIARI \& FIGUEIREDO, 2012). Tal situação tem se tornado um grande desafio para as políticas de saúde pública, pois há uma carência de assistência social e de serviços de atenção integral aos indivíduos, às famílias e 
comunidade que enfrentam esse problema. O controle dessa endemia pode ocorrer de forma progressiva com a ampliação desses serviços e a sua integração ao PNCT, sendo uma iniciativa pública fundamental (REIS et al., 2013).

Quanto à baciloscopia, a frequência em que o exame foi realizado $(76,1 \%)$ se mostrou inferior ao estudo realizado em Teresina, com 82,1\% (COELHO et al., 2010), porém semelhante ao estudo descrito na cidade de Salvador-BA (XAVIER \& BARRETO, 2007). O percentual de $54,2 \%$ de positividade encontrado é inferior aos $60 \%$ a $80 \%$ preconizados pelo Ministério da Saúde, que o exame permite detectar (BRASIL, 2011; COUTINHO et al., 2012).

Sobre a forma de entrada, $85,7 \%$ dos casos entraram como caso novo. Resultados similares foram encontrados em outros trabalhos epidemiológicos (COUTINHO et al., 2012; PEREIRA et al., 2015). Os casos novos refletem a dinâmica com que os casos aparecem na população, por isso é fundamental a observância e o controle da tuberculose, que tem como ferramentas a detecção precoce dos casos novos e o tratamento eficaz da doença. A taxa de transferência dos casos estudados está relacionada ao fato do diagnóstico ser feito em outro município e o tratamento ser realizado de forma oportuna no município de Imperatriz-MA (MOREIRA et al., 2007).

No que se refere à condição de encerramento, destaca-se que $88,7 \%$ tiveram alta por cura, taxa superior a encontrada em estudo (ALVES et al., 2014) realizado no município de Santa Maria-RS. No combate à tuberculose, o Ministério da Saúde determinou o TDO e estipulou metas para o alcance de $85 \%$ de cura e a diminuição das taxas de abandono do esquema medicamentoso para $5 \%$ ou menos. Apesar das metas de cura e abandono terem sido alcançadas, o TDO do município não foi compatível com o preconizado pelo Ministério da Saúde, que estabelece uma cobertura de $100 \%$ (BRASIL, 2011) perfazendo uma cobertura de somente $58,5 \%$ da população acometida pela doença, porém com resultado similiar ao estudo de PEREIRA et al., (2015).

Estudos indicam que o TDO tem sido decisivo para se alcançar resultados satisfatórios (ALVES et al., 2014; YAMAMURA et al., 2014). E isso pode garantir uma maior adesão dessa população, porém ainda não é suficiente para resolver o problema que, dentro de uma visão de promoção de saúde, poderia ocorrer através da negociação entre os gestores da saúde e educação para maiores investimentos em programas que já existem no município (ALVES et al., 2014).

\section{CONCLUSÃO}

Os resultados obtidos permitiram conhecer os aspectos da tuberculose em Imperatriz-MA, e perceber que de um modo geral houve um declínio dos casos da doença no município entre os anos 2009 e 2012 e um aumento em 2013. Além disso, o perfil epidemiológico da doença no município mostra-se compatível com as observações nacionais de outros serviços.

Os resultados desse estudo fornecem subsídios para a tomada de ações, a fim de que os grupos de maior risco, entre eles pacientes com agravos associados e aposentados, possam ter uma abordagem especial dos programas de controle da doença, culminando em menor número de notificações de tuberculose no município de Imperatriz-MA.

São necessários maiores esforços empreendidos na intensificação à prevenção, com ações de educação em saúde permanentes, incluindo programas 
de aumento do nível de escolarização das pessoas mais vulneráveis à doença, e também às ações de vigilância epidemiológica. O aperfeiçoamento do sistema de informações sobre a doença e uma integração mais eficaz dos programas de controle da AIDS e da tuberculose, além de estratégias que viabilizem maior acesso dos homens aos serviços de saúde.

\section{REFERÊNCIAS}

ALVES, R.H.; REIS, D.C.; VIEGAS, A.M.; NEVES, J.A.C.; ALMEIDA,T.A.C. Epidemiologia da tuberculose no município de Contagem, Minas Gerais, Brasil, entre 2002 e 2011. Revista de Epidemiologia e Controle de Infecção. v. 4, n. 2, p:146$153.2014 . \quad$ Disponível em:

online.unisc.br/seer/index.php/epidemiologia/article/view/4411>.

BRASIL. Ministério da Saúde. Secretaria de Vigilância em Saúde. Departamento de Vigilância Epidemiológica. Doenças infecciosas e parasitárias: guia de bolso. Brasília (DF), p: 412-428. 2010.

BRASIL. Ministério da Saúde. Secretaria de vigilância em saúde. Manual de Recomendações para o controle da tuberculose no Brasil. Programa Nacional de Controle da Tuberculose. Brasília (DF), p. 75-84. 2011.

BRASIL. Informações de Saúde. Sistema de Informação de Agravos de Notificação - SINAN [Internet]. Disponível em: <http://dtr2004.saude.gov.br/sinanweb/>. 2014a.

BRASIL. Ministério da Saúde. Secretaria de Vigilância em Saúde. Boletim Epidemiológico - Especial Tuberculose. Brasília (DF), v. 44, n. 2, p: 1-13. 2014b.

BRASIL. Ministério da Saúde. Secretaria de Vigilância em Saúde. Boletim Epidemiológico. Brasília (DF), v. 46, n. 9. 2015a.

BRASIL. Ministério da Saúde. Departamento de Atenção Básica (DAB). 2015b. [Internet]. Disponível em:

<http://dab.saude.gov.br/portaldab/sala_apoio_gestao_estrategica.php. 2015>.

CALIARI, J.; FIGUEIREDO, R.M. Tuberculosis: patient profile, service flowchart, and nurses' opinions. Acta Paulista de Enfermagem. v. 25, n. 1, p. 43-47, 2012.

Disponível em: <http://dx.doi.org/10.1590/S0103-21002012000100008>. doi: 10.1590/S0103-21002012000100008

CAVALCANTE, E.F.O; SILVA, D.G.V. Perfil de pessoas acometidas por tuberculose. Revista Rene. v. 14, n. 4, 2013. Disponível em:

< http://www.redalyc.org/articulo.oa?id=324028459008>.

COÊLHO, D. M. M.; VIANA, R. L.; MADEIRA, C. A.; FERREIRA, L. O. C.; CAMPELO, V. Perfil epidemiológico da tuberculose no Município de Teresina-PI, no período de 1999 a 2005. Epidemiologia e Serviços de Saúde. v. 19, n. 1, 2010. Disponível em: <http://dx.doi.org/10.5123/S1679-49742010000100005>. doi: 10.5123/S1679-49742010000100005. 
COUTINHO, L.A.S.A.; OLIVEIRA, D.S.; SOUZA, G.F.; FERNANDES FILHO, G.M.S.; SARAIVA, M.G. Perfil Epidemiológico da Tuberculose no Município de João Pessoa-PB, entre 2007-2010. Revista Brasileira de Ciências da Saúde, v. 16, n. 1, p. 35-42, 2012. Disponível em:

<http://periodicos.ufpb.br/ojs/index.php/rbcs/article/view/10172/7085>.

DAMACENO, A.N.; RAMOS, L.S.; WEILLER, TH. Perfil clínico epidemiológico de portadores de tuberculose em Santa Maria (2001 a 2012). Espaço para a Saúde. v.15, n. 4, p: 34-41, 2014. Disponível em:

<http://www.uel.br/revistas/uel/index.php/espacoparasaude/article/view/19048>.

DURANS, J.J.F.; SÁ, E.M; PEREIRA,L.F.B.; SOARES, D.L.; OLIVEIRA, P.S.; AQUINO, D.M.C.; CALDAS, A.J.M. Perfil clínico e sociodemográfico de pacientes que abandonaram o tratamento de tuberculose no município de São Luís - MA. Revista de Pesquisa em Saúde. v. 14, n. 3, p: 175-8, 2013. Disponível em: <http://www.periodicoseletronicos.ufma.br/index.php/revistahuufma/article/view/2794 $>$.

GUIMARAES, R.M.; LOBO, A.P.; SIQUEIRA, E.A.; BORGES, T.F.F.; MELO, S.C.C. Tuberculose, HIV e pobreza: tendência temporal no Brasil, Américas e mundo. Jornal Brasileiro de Pneumologia. v. 38, n. 4, p. 511-517, 2012. Disponível em: <http://dx.doi.org/10.1590/S1806-37132012000400014>. doi: 10.1590/S1806-37132012000400014.

HINO, P.; CUNHA, T.N.; VILLA, T.C.S.; SANTOS, C.B. Revista Ciência \& Saúde Coletiva. v. 16, supl. 1, p. 1295-1301, 2011. Disponível em <http://dx.doi.org/10.1590/S1413-81232011000700063>. doi: 10.1590/S141381232011000700063.

INSTITUITO BRASILEIRO DE GEOGRAFIA E ESTATÍTICA (IBGE). Censo Demográfico 2010. [internet]. Disponível em:<http://www.ibge.gov.br/home/estatistica/populacao/censo2010/. 2010.

LIN, Y.; LI, L.; MI, F.; DU, J.; DONG, Y.; LI, Z.; QI, W.; ZHAO, X.; CUI, Y.; HOU, F.; ZACHARIAH, R.; KAPUR, A.; LÖNNROTH, K.; HARRIES, A.D. Screening patients with diabetes mellitus for tuberculosis in China. Tropical Medicine \& International Health. v. 17, n. 10, p:1302-08. doi: 10.1111/j.1365-3156.2012.03069.x.

LOPES, T.; GOMES, C.; DIOGO, N. Tuberculosis unit: study of 10 years of activity (1999-2009). Revista Portuguesa de Pneumologia. v. 17, n. 6, p:266-271. 2011. doi: 10.1016/j.rppneu.2011.06.008.

MACIEL, E.L.N. Estratégias da agenda pós-2015 para o controle da tuberculose no Brasil: desafios e oportunidades. Epidemiologia e Serviços de Saúde. Brasília, v. 25, n. 2, p. 423-426, 2016. Disponível em: < $<$ http://www.scielo.br/scielo.php?script=sci_arttext\&pid=S2237-

96222016000200423\&Ing=en\&nrm=iso>. $\quad$ doi: http://dx.doi.org/10.5123/S1679- 
49742016000200021.

MASCARENHAS, M.D.M.; ARAUJO, L.M.; GOMES, K.R.O. Perfil epidemiológico da tuberculose entre casos notificados no Município de Piripiri, Estado do Piauí, Brasil Epidemiologia e Serviços de Saúde. v. 14, n. 1,2005. Disponível em <http://dx.doi.org/10.5123/S1679-49742005000100002>. doi: 10.5123/S167949742005000100002.

MENDONCA, S.A.; FRANCO, S.C. Avaliação do risco epidemiológico e do desempenho dos programas de controle de tuberculose nas Regiões de Saúde do estado de Santa Catarina, 2003 a 2010. Epidemiologia e Serviços de Saúde. v. 24, n. 1, p. 59-70, 2015. Disponível em: <http://dx.doi.org/10.5123/S167949742015000100007>. doi:10.5123/S1679-49742015000100007.

MOREIRA, M.A.C.; BELLO, A.S.; ALVES, M.R.L.; SILVA, M.V.; LORUSSO, V. Avaliação da notificação no Distrito Federal de casos de tuberculose residentes em dez municípios goianos do entorno e análise da incidência de tuberculose nestas localidades. Jornal Brasileiro de Pneumologia. v. 33, n. 3, p. 301-310, 2007.

Disponível em: <http://dx.doi.org/10.1590/S1806-37132007000300012>. doi: 10.1590/S1806-37132007000300012.

ONOZAKI, I.; LAW, I.; SISMANIDIS, C.; ZIGNOL, M.; GLAZIOU, P.; FLOYD, K. National tuberculosis prevalence surveys in Asia, 1990-2012: an overview of results and lessons learned. Tropical Medicine \& International Health. v. 20, n. 9, p. 11281145, 2015. doi: 10.1111/tmi.12534.

OROFINO, R.L.; BRASIL, P.E.A.; TRAJMAN, A.; SCHMALTZ, C.A.S.; DALCOLMO, M.; ROLLA, V.C. Preditores dos desfechos do tratamento da tuberculose. Jornal Brasileiro de Pneumologia. v. 38, n. 1, p. 88-97, 2012. Disponível em: <http://dx.doi.org/10.1590/S1806-37132012000100013>. doi: 10.1590/S180637132012000100013.

PEREIRA, J.C.; SILVA, M.R.; COSTA, R.R.; GUIMARÃES, M.D.C.; LEITE, I.C.G. Perfil e seguimento dos pacientes com tuberculose em município prioritário no Brasil. Revista de Saúde Pública. v. 49,2015. Disponível em <http://dx.doi.org/10.1590/S0034-8910.2015049005304> doi: 10.1590/S00348910.2015049005304.

REIS, D.C.; ALMEIDA, T.A.C.; QUITES, H.F.O.; SAMPAIO, M.M. Perfil epidemiológico da tuberculose no Município de Belo Horizonte (MG), no período de 2002 a 2008. Revista Brasileira de Epidemiologia. v. 16, n. 3, p. 592-602, 2013. Disponível em:<http://dx.doi.org/10.1590/S1415-790X2013000300004>. doi: 10.1590/S1415-790X2013000300004.

SAGILI, K.D.; SATYANARAYANA, S.; CHADHA, S.S. Is Knowledge Regarding Tuberculosis Associated with Stigmatising and Discriminating Attitudes of General Population towards Tuberculosis Patients? Findings from a Community Based Survey in 30 Districts of India. PLoS One. Fev 1;11(2):e0147274. 2016. doi: 
10.1371/journal.pone.0147274.

SANTOS NETO, M.; SILVA, F.L.; SOUSA, K.R.; YAMAMURA, M.; POPOLIN, M.P.; ARCÊNCIO, R.A. Perfil clínico e epidemiológico e prevalência da coinfecção tuberculose/HIV em uma regional de saúde no Maranhão. Jornal Brasileiro de Pneumologia. v. 38, n. 6, p. 724-732, 2012. Disponível em:< <http://dx.doi.org/10.1590/S1806-37132012000600007>. doi: 10.1590/S180637132012000600007.

SANTOS, T.M.M.G.; NOGUEIRA, L.T.; ARCENCIO, R.A. Atuação de profissionais da Estratégia Saúde da Família no controle da tuberculose. Acta Paulista de Enfermagem. v. 25, n. 6, p. 954-961, $2012 . \quad$ Disponível em:<http://dx.doi.org/10.1590/S0103-21002012000600020>. doi: 10.1590/S010321002012000600020

SILVA, C.C.A.V.; ANDRADE, M.S.; CARDOSO, M.D. Fatores associados ao abandono do tratamento de tuberculose em indivíduos acompanhados em unidades de saúde de referência na cidade do Recife, Estado de Pernambuco, Brasil, entre 2005 e 2010. Epidemiologia e Serviços de Saúde. v. 22, n. 1, 2013. Disponível em <http://dx.doi.org/10.5123/S1679-49742013000100008>. doi: 10.5123/S167949742013000100008.

SILVA, P.F.; MOURA, G.S.; CALDAS, A.J.M. Fatores associados ao abandono do tratamento da tuberculose pulmonar no Maranhão, Brasil, no período de 2001 a 2010. Cadernos de Saúde Pública, Rio de Janeiro, v. 30, n. 8, p. 1745-1754, 2014. Disponível em: <http://dx.doi.org/10.1590/0102-311X00124513>. doi: 10.1590/0102-311X00124513

XAVIER, M.I.M.; BARRETO, M.L. Tuberculose na cidade de Salvador, Bahia, Brasil: o perfil na década de 1990. Cadernos de Saúde Pública, v. 23, n. 2, p. 445453, 2007. Disponível em: <http://dx.doi.org/10.1590/S0102-311X2007000200021. doi: 10.1590/S0102-311X2007000200021.

YAMAMURA, M.; SANTOS NETO, M.; FREITAS, I.M.; RODRIGUES, L.B.B.; POPOLIN, M.P.; UCHOA, S.A.C.; FRONTEIRA, I.; ARCÊNCIO, R.A. Tuberculose e iniquidade social em saúde: uma análise ecológica utilizando técnicas estatísticas multivariadas, São Paulo, Brasil. Revista Panamericana de Salud Pública, v. 35, n. 4, p. 270-277, 2014. Disponível em:

<http://www.scielosp.org/scielo.php?script=sci_arttext\&pid=S1020-

$49892014000400006 \&$ Ing=en\&nrm=iso>. 